\title{
Spinor-helicity variables for cosmological horizons in de Sitter space
}

\author{
Adrian David, ${ }^{1, *}$ Nico Fischer, ${ }^{2, \dagger}$ and Yasha Neiman ${ }^{1, *}$ \\ ${ }^{1}$ Okinawa Institute of Science and Technology, 1919-1 Tancha, Onna-son, Okinawa 904-0495, Japan \\ ${ }^{2}$ Friedrich Schiller University Jena, 07737 Jena, Germany
}

(Received 10 June 2019; published 7 August 2019)

\begin{abstract}
We consider massless fields of arbitrary spin in de Sitter space. We introduce a spinor-helicity formalism, which encodes the field data on a cosmological horizon. These variables reduce the free S-matrix in an observer's causal patch, i.e., the evolution of free fields from one horizon to another, to a simple Fourier transform. We show how this result arises via twistor theory, by decomposing the horizon $\leftrightarrow$ horizon problem into a pair of (more symmetric) horizon $\leftrightarrow$ twistor problems.
\end{abstract}

DOI: 10.1103/PhysRevD.100.045005

\section{INTRODUCTION}

In field theory on flat spacetime, the S-matrix between past and future infinity is an object of fundamental importance. For massless theories such as Yang-Mills and general relativity (GR), the spinor-helicity formalism [1] has emerged as the ideal language [2] for studying the S-matrix (with the exception of some highly symmetric cases, in which twistor language is superior [3-5]). Since our Universe appears to have a positive cosmological constant, it is of great theoretical interest to study the "S-matrix" in a static (i.e., observable) patch of de Sitter space, with an observer's past and future horizons in the roles of past/future infinity. So far, there has been remarkably little work on this problem. Instead, the main focus of theoretical attention in de Sitter space has been with correlations on its conformal boundary [6-8], which are unobservable in a true asymptotic de Sitter space (but become observable in approximate, temporary de Sitter scenarios such as inflation).

In this paper, we take some first steps toward the de Sitter S-matrix. First, we encode the lightlike field data on a cosmological horizon in terms of spinor-helicity variables, equivalent to those introduced in [7] for the Poincare patch (see also the constructions for anti-de Sitter, in the Poincare patch [9] and in stereographic coordinates [10]). Then, in our main result, we relate the spinor-helicity variables associated with two cosmological horizons (and thus two

\footnotetext{
*adrian.david@oist.jp †nico.fischer@uni-jena.de "yashula@icloud.com
}

Published by the American Physical Society under the terms of the Creative Commons Attribution 4.0 International license. Further distribution of this work must maintain attribution to the author(s) and the published article's title, journal citation, and DOI. Funded by SCOAP .
Poincare patches) to obtain the free S-matrix in the static patch for massless fields of any spin. Our formalism and result provide a plausible starting point for efficiently including the effects of interactions in future work.

\section{GEOMETRIC SETUP}

De Sitter space is best described as a hyperboloid of unit spacelike radius embedded in flat $4+1 \mathrm{~d}$ spacetime,

$$
d S_{4}=\left\{x^{\mu} \in \mathbb{R}^{1,4} \mid x_{\mu} x^{\mu}=1\right\} .
$$

We will use lightcone coordinates $x^{\mu}=(u, v, \mathbf{r})$ for $\mathbb{R}^{1,4}$, where $\mathbf{r}$ is an $\mathbb{R}^{3}$ vector, and the metric is $d x_{\mu} d x^{\mu}=$ $-d u d v+\mathbf{d} \mathbf{r}^{2}$. These coordinates are adapted to a de Sitter observer, whose initial and final horizons are defined by $(u=0, v<0)$ and $(u>0, v=0)$, respectively. The horizons' spatial section is the two-sphere $S_{2}$ of unit vectors $\mathbf{r}^{2}=1$. The tangent space of this $S_{2}$ at a point $\mathbf{r}$ can be spanned by a complex null basis $(\mathbf{m}, \overline{\mathbf{m}})$,

$$
\mathbf{m} \cdot \mathbf{r}=0 ; \quad \mathbf{m}^{2}=0 ; \quad \mathbf{m} \times \overline{\mathbf{m}}=-i \mathbf{r} .
$$

This basis is defined up to phase rotations $(\mathbf{m}, \overline{\mathbf{m}}) \rightarrow$ $\left(e^{i \theta} \mathbf{m}, e^{-i \theta} \overline{\mathbf{m}}\right)$, which describe $S O(2)$ rotations of the $S_{2}$ tangent space. In our setup, these rotations will play the role of the massless fields' little group.

Vectors in $d S_{4}$ are simply $\mathbb{R}^{1,4}$ vectors constrained to the tangent space of the hyperboloid (1). Spinors in $d S_{4}$ can be constructed similarly from embedding-space spinors (see e.g., [11]), but we will not need that construction here. For the statement of our main result, it will suffice to introduce the two-component spinors $\psi^{\alpha}$ of spatial $S O(3)$ rotations. The antisymmetric metric on $S O(3)$ spinors is $\epsilon_{\alpha \beta}$, with inverse $\epsilon^{\alpha \gamma} \epsilon_{\beta \gamma}=\delta_{\beta}^{\alpha}$. We raise and lower indices via $\psi_{\alpha}=\epsilon_{\alpha \beta} \psi^{\beta}$. We denote the Pauli matrices by $\boldsymbol{\sigma}_{\beta}^{\alpha}$. 
Spinors have a complex conjugation $\psi^{\alpha} \rightarrow \bar{\psi}^{\alpha} \rightarrow-\psi^{\alpha}$, under which $\epsilon_{\alpha \beta}$ is real but $\boldsymbol{\sigma}_{\beta}^{\alpha}$ is imaginary.

For the derivation of our main result, we will also need the four-component spinors of the $\mathbb{R}^{1,4}$ embedding space, i.e., the twistors of $d S_{4}$. These can be constructed as pairs of $S O(3)$ spinors (see e.g., [12]),

$$
Y^{a}=\left(\begin{array}{c}
\lambda_{\alpha} \\
i \bar{\mu}^{\alpha}
\end{array}\right)
$$

where the $i$ and complex conjugation on the second spinor are for later convenience. The $S O(1,4)$ spinor index $a$ is lowered via $Y_{a}=\left(-i \bar{\mu}^{\alpha}, \lambda_{\alpha}\right)$. Complex conjugation is inherited directly from that of the $S O(3)$ spinors. The $\mathbb{R}^{1,4}$ gamma matrices $\gamma_{\mu}=\left(\gamma_{u}, \gamma_{v}, \boldsymbol{\gamma}\right)$ can be written in $2 \times 2$ block notation as

$$
\left(\gamma_{\mu}\right)_{b}^{a}=\left(\left(\begin{array}{cc}
0 & 0 \\
-\epsilon^{\alpha \beta} & 0
\end{array}\right),\left(\begin{array}{cc}
0 & \epsilon_{\alpha \beta} \\
0 & 0
\end{array}\right),\left(\begin{array}{cc}
-i \boldsymbol{\sigma}_{\alpha}^{\beta} & 0 \\
0 & -i \boldsymbol{\sigma}_{\beta}^{\alpha}
\end{array}\right)\right) .
$$

We will sometimes omit both $S O(3)$ and $S O(1,4)$ spinor indices. In a product, this will imply bottom-to-top index contraction.

\section{FIELD DATA ON THE HORIZON}

We consider the free massless field equation for a totally symmetric, double-traceless spin-s gauge potential $h_{\mu_{1} \ldots \mu_{s}}$ in $d S_{4}$ [13],

$$
\begin{gathered}
\left(\square+2\left(s^{2}-1\right)\right) \phi_{\mu_{1} \ldots \mu_{s}}-s \nabla_{\rho} \nabla_{\left(\mu_{1}\right.} \phi_{\left.\mu_{2} \ldots \mu_{s}\right)}^{\rho} \\
+\frac{s(s-1)}{2} \nabla_{\left(\mu_{1}\right.} \nabla_{\mu_{2}} \phi_{\left.\mu_{3} \ldots \mu_{s}\right) \nu}^{\nu}=0,
\end{gathered}
$$

with a gauge symmetry $\delta \phi_{\mu_{1} \ldots \mu_{s}}=\nabla_{\left(\mu_{1}\right.} \Lambda_{\left.\mu_{2} \ldots \mu_{s}\right)}$ for totally symmetric, traceless $\Lambda_{\mu_{1} \ldots \mu_{s-1}}$. The cases $s=0,1,2$ describe the conformally coupled massless scalar, the Maxwell equations, and linearized GR, respectively. In the scalar case, the field's value $\phi(u, 0, \mathbf{r})$ on e.g., the final horizon constitutes good boundary data for the field equation $(\square-2) \phi=0$. For nonzero spin, good boundary data consists of one complex scalar component for the right-handed helicity, and its complex conjugate for the left-handed one; see e.g., [14-16] for the standard construction in flat spacetime and [17] for a general discussion in terms of field strengths. In our present context, we can fix a gauge such that $\phi_{\mu_{1} \ldots \mu_{s}}$ on the horizon has only spatial components $\phi_{i_{1} \ldots i_{s}}$. Here, the $i_{k}$ 's are $\mathbb{R}^{3}$ indices, which must be tangent to the $d S_{4}$ hyperboloid, and thus to the $S_{2}$ horizon section. The horizon boundary data are then given by the traceless part of this $\phi_{i_{1} \ldots i_{s}}$. Using the complex basis (2) for the $S_{2}$ tangent space, we can reduce this traceless part to a pair of scalars,

$$
\begin{aligned}
\phi^{(s)}(u, \mathbf{r} ; \mathbf{m}) & =m^{i_{1}} \ldots m^{i_{s}} \phi_{i_{1} \ldots i_{s}}(u, 0, \mathbf{r}), \\
\phi^{(-s)}(u, \mathbf{r} ; \mathbf{m}) & =\bar{m}^{i_{1}} \ldots \bar{m}^{i_{s}} \phi_{i_{1} \ldots i_{s}}(u, 0, \mathbf{r}) .
\end{aligned}
$$

These respectively describe fields of helicity $\pm s$ and carry weight $\pm s$ under the phase rotation $(\mathbf{m}, \overline{\mathbf{m}}) \rightarrow$ $\left(e^{i \theta} \mathbf{m}, e^{-i \theta} \overline{\mathbf{m}}\right)$. The symplectic form for the horizon data (5) reads

$$
\Omega\left[\delta \phi_{1}, \delta \phi_{2}\right]=\sum_{h= \pm s} \int d u \int_{S_{2}} d^{2} \mathbf{r} \delta \phi_{2}^{(h)} \frac{\overleftrightarrow{\partial}}{\partial u} \delta \phi_{1}^{(-h)},
$$

where we sum over the two helicities $\phi^{( \pm s)}$ in the spinning case, or over just one helicity $\phi^{(0)} \equiv \phi$ in the scalar case.

The boundary data on the initial horizon can be encoded in the same way. Replacing the null time $u$ with $v$, and noticing that the helicity associated with $(\mathbf{m}, \overline{\mathbf{m}})$ is now reversed, we write

$$
\begin{aligned}
\tilde{\phi}^{(s)}(v, \mathbf{r} ; \mathbf{m}) & =\bar{m}^{i_{1}} \ldots \bar{m}^{i_{s}} \phi_{i_{1} \ldots i_{s}}(0, v, \mathbf{r}), \\
\tilde{\phi}^{(-s)}(v, \mathbf{r} ; \mathbf{m}) & =m^{i_{1}} \ldots m^{i_{s}} \phi_{i_{1} \ldots i_{s}}(0, v, \mathbf{r}) .
\end{aligned}
$$

Finally, it is useful to define the gauge-invariant field strength data corresponding to the gauge potential data [Eqs. (5) and (7)],

$$
\begin{aligned}
& C^{( \pm s)}(u, \mathbf{r} ; \mathbf{m})=\frac{\partial^{s}}{\partial u^{s}} \phi^{( \pm s)}(u, \mathbf{r} ; \mathbf{m}), \\
& \tilde{C}^{( \pm s)}(v, \mathbf{r} ; \mathbf{m})=\frac{\partial^{s}}{\partial v^{s}} \tilde{\boldsymbol{\phi}}^{( \pm s)}(v, \mathbf{r} ; \mathbf{m}) .
\end{aligned}
$$

\section{THE S-MATRIX PROBLEM}

For our purposes, the S-matrix problem in de Sitter space is to relate the gauge-invariant field data (8) on the final horizon to the corresponding data (9) on the initial one. This statement of the problem, which will be more convenient for us, is slightly more general than what is usually termed the S-matrix. Usually, one would relate the quantum states obtained by acting with the fields on some vacuum; by focusing on the fields themselves, we avoid committing to a particular vacuum state. We will ignore here any subtleties related to zero-frequency modes, i.e., to the horizons' lower-dimensional boundaries (either at asymptotic infinity or at the horizons' $S_{2}$ intersection). In other words, we will be dealing with the "hard part" of the S-matrix.

For free fields, one can find the S-matrix by "brute force", using the general technique for linear hyperbolic equations. Essentially, the value of a massless field at some final horizon point is determined by the intersection of that point's past lightcone with the initial horizon. Thus, for e.g., the scalar field, we have 


$$
\phi(u, 0, \mathbf{r})=\left.\frac{1}{\pi u} \int_{S_{2}} d^{2} \mathbf{r}^{\prime} \frac{\partial \phi\left(0, v, \mathbf{r}^{\prime}\right)}{\partial v}\right|_{v=2\left(\mathbf{r} \cdot \mathbf{r}^{\prime}-1\right) / u},
$$

which can be obtained from the general formula,

$$
\phi(u, 0, \mathbf{r})=\int d v \int_{S_{2}} d^{2} \mathbf{r}^{\prime} \phi\left(0, v, \mathbf{r}^{\prime}\right) \frac{\overleftrightarrow{\partial}}{\partial v} G\left(u, 0, \mathbf{r} ; 0, v, \mathbf{r}^{\prime}\right),
$$

in which $G\left(x^{\mu} ; x^{\prime \mu}\right)=(-1 / 4 \pi) \delta\left(x_{\mu} x^{\prime \mu}-1\right) \theta\left(u-u^{\prime}\right)$ is a causal Green's function in $d S_{4}$. For the analogous general treatment of nonzero spin, see [17]. In fact, the end result (10) holds not only in the static patch $(u>0, v<0)$, but also for the horizons' entire extent $u, v \in \mathbb{R}$, which includes the antipodal patch $(u<0, v>0)$. In the rest of the paper, we will avoid specifying the range of $u, v$, and our formulas will apply equally well to both $(u>0, v<0)$ and $u, v \in \mathbb{R}$. While the $(u>0, v<0)$ case is linked more directly to observable physics, our formulas "live more naturally" in the more global context $u, v \in \mathbb{R}$.

Despite its simplicity, Eq. (10) is not quite satisfactory. Since it does not make explicit contact with the horizons' symmetries, it is unlikely as a useful starting point for interacting calculations.

What, then, are the relevant symmetries? Naively, they are the subgroup of $d S_{4}$ isometries that preserves both horizons. These are the static-patch time translations $(u, v) \rightarrow\left(e^{t} u, e^{-t} v\right)$ and the $S O(3)$ rotations of $\mathbf{r}$. These symmetries encourage one to work in terms of frequencies and spherical harmonics. The S-matrix for the free scalar in this basis was found in $[18,19]$. However, spherical harmonics are rather unpleasant, so the generalization to interacting theories again does not seem promising. Below, we will describe a different basis for the S-matrix, which replaces spherical harmonics with plane waves, by fixing only one horizon at a time.

\section{POINCARE MOMENTUM AND SPINOR-HELICITY}

Instead of fixing both horizons, let us consider the residual symmetry from fixing just e.g., the final one. This is the symmetry of the Poincare patch: the translations, rotations, and dilatations of $\mathbb{R}^{3}$. On the horizon, the rotations act on $\mathbf{r} \in S_{2}$ in the obvious way, the dilatations rescale $u$, while a translation by a vector a shifts the light rays according to $u \rightarrow u-2 \mathbf{a} \cdot \mathbf{r}$. A fixed momentum $\mathbf{p}$ with respect to these translations describes two modes on the horizon,

$$
\begin{aligned}
& \text { Positive frequency: } \delta^{2}\left(\mathbf{r},+\frac{\mathbf{p}}{|\mathbf{p}|}\right) e^{-i|\mathbf{p}| u / 2} \\
& \text { Negative frequency: } \delta^{2}\left(\mathbf{r},-\frac{\mathbf{p}}{|\mathbf{p}|}\right) e^{+i|\mathbf{p}| u / 2}
\end{aligned}
$$

These modes are waves with frequency $\pm|\mathbf{p}| / 2$ with respect to the null time $u$, supported on an antipodal pair of light rays $\mathbf{r}= \pm \mathbf{p} /|\mathbf{p}|$.

Let us now define spinor-helicity variables $\lambda_{\alpha}, \bar{\lambda}_{\alpha}$ as the spinor square root of $\mathbf{p}$, such that $\mathbf{p}=\bar{\lambda} \sigma \lambda$. The corresponding positive-frequency mode will be a wave supported at $\mathbf{r}=(\bar{\lambda} \sigma \lambda) /(\bar{\lambda} \lambda)$, with frequency $\bar{\lambda} \lambda / 2$ with respect to $u$. Moreover, we can use $\mathbf{m}=i(\lambda \sigma \lambda) /(\sqrt{2} \bar{\lambda} \lambda)$ and its complex conjugate $\overline{\mathbf{m}}$ as the complex null basis (2) for the $S_{2}$ tangent space. Thus, we package the positivefrequency part of the horizon field data (5) into spinor functions $f^{( \pm s)}\left(\lambda_{\alpha}, \bar{\lambda}_{\alpha}\right)$ as follows:

$f^{( \pm s)}\left(\lambda_{\alpha}, \bar{\lambda}_{\alpha}\right)=\int d u e^{i(\bar{\lambda} \lambda) u / 2} \phi^{( \pm s)}\left(u, \frac{\bar{\lambda} \sigma \lambda}{\bar{\lambda} \lambda} ; \frac{i \lambda \sigma \lambda}{\sqrt{2} \bar{\lambda} \lambda}\right)$.

These spinor functions have the following manifest symmetries:

(i) By construction, they have momentum $\bar{\lambda} \sigma \lambda$ under the Poincare-patch translations $u \rightarrow u-2 \mathbf{a} \cdot \mathbf{r}$.

(ii) Under Poincare-patch dilatations, i.e., static-patch time translations $(u, v) \rightarrow\left(e^{t} u, e^{-t} v\right)$, they scale as $f^{( \pm s)}(\lambda, \bar{\lambda}) \rightarrow e^{t} f^{( \pm s)}\left(e^{t / 2} \lambda, e^{t / 2} \bar{\lambda}\right)$.

(iii) The field's helicity $\pm s$ is encoded in the scaling relation $f^{( \pm s)}\left(e^{i \theta / 2} \lambda, e^{-i \theta / 2} \bar{\lambda}\right)=e^{ \pm i s \theta} f^{( \pm s)}(\lambda, \bar{\lambda})$.

In fact, along with the more obvious $S O(3)$ rotations, these symmetries uniquely determine the encoding (13) of horizon data into spinor functions, up to a prefactor that can only depend on helicity. As a corollary, we conclude that this spinor-helicity formalism must coincide with the one constructed from a different point of view in [7].

As is frequently useful in the spinor-helicity formalism [20], we can analytically continue to complex momenta by making the two spinors $(\lambda, \bar{\lambda})$ independent. As a special case, by analytically continuing $(\lambda, \bar{\lambda}) \rightarrow(i \lambda, i \bar{\lambda})$, we obtain the negative-frequency modes,

$$
f^{( \pm s)}(i \lambda, i \bar{\lambda})=\left(f^{(\mp s)}(\lambda, \bar{\lambda})\right)^{*} .
$$

In these variables, the field's symplectic form (6) reads simply

$$
\begin{aligned}
\Omega\left[\delta f_{1}, \delta f_{2}\right]= & \frac{1}{i} \sum_{h= \pm s} \int \frac{d^{2} \lambda d^{2} \bar{\lambda}}{(2 \pi i)^{2}} \\
& \times\left(\delta f_{1}^{(h)}(\lambda, \bar{\lambda}) \delta f_{2}^{(-h)}(i \lambda, i \bar{\lambda})-(1 \leftrightarrow 2)\right),
\end{aligned}
$$

where $d^{2} \lambda$ is the spinor measure $\epsilon_{\alpha \beta} d \lambda^{\alpha} d \lambda^{\beta} / 2$.

The field data (7) on the initial horizon can be treated in the same way. Thus, the positive-frequency modes are encoded as

$$
\tilde{f}^{( \pm s)}\left(\mu^{\alpha}, \bar{\mu}^{\alpha}\right)=\int d v e^{i(\bar{\mu} \mu) v / 2} \tilde{\phi}^{( \pm s)}\left(v, \frac{\bar{\mu} \boldsymbol{\sigma} \mu}{\bar{\mu} \mu} ; \frac{i \mu \sigma \mu}{\sqrt{2} \bar{\mu} \mu}\right) .
$$


The negative-frequency modes can again be obtained by analytic continuation, as in (14). Note that $\mu^{\alpha}$ is now the square root of momentum in a different Poincare coordinate patch-the one associated with the initial horizon.

\section{THE FREE S-MATRIX}

In the framework of the previous section, finding the S-matrix means relating the spinor functions $f^{( \pm s)}(\lambda, \bar{\lambda})$ and $\tilde{f}^{( \pm s)}(\mu, \bar{\mu})$. Our paper's main result (to be derived in the next section) is that the free-field answer is simply a Fourier transform,

$f^{( \pm s)}\left(\lambda_{\alpha}, \bar{\lambda}_{\alpha}\right)=\int \frac{d^{2} \mu d^{2} \bar{\mu}}{(2 \pi i)^{2}} \tilde{f}^{( \pm s)}\left(\mu^{\alpha}, \bar{\mu}^{\alpha}\right) e^{i\left(\lambda_{\alpha} \mu^{\alpha}+\bar{\lambda}_{\alpha} \bar{\mu}^{\alpha}\right)}$.

This formula can be unpacked explicitly in terms of the horizon field data (8) and (9), giving

$$
\begin{aligned}
& C^{( \pm s)}(u, \mathbf{r} ; \mathbf{m})=\frac{2^{s}}{\pi u^{2 s+1}} \\
& \quad \times\left.\int_{S_{2}} d^{2} \mathbf{r}^{\prime}\left(1-\mathbf{r} \cdot \mathbf{r}^{\prime}\right)^{s} \frac{\partial \tilde{C}^{( \pm s)}\left(v, \mathbf{r}^{\prime} ; \mathbf{m}^{\prime}\right)}{\partial v}\right|_{v=2\left(\mathbf{r} \cdot \mathbf{r}^{\prime}-1\right) / u},
\end{aligned}
$$

which reproduces the spin-0 result (10) as a special case. The phase of the null tangent vector $\mathbf{m}^{\prime}$ in (18) is fixed via

$$
2 \mathbf{m} \cdot \mathbf{m}^{\prime}=1-\mathbf{r} \cdot \mathbf{r}^{\prime} .
$$

The explicit S-matrix (18) can be derived from the main result (17) and the definitions (8), (9), (13), (16), via mostly straightforward integrals. The two nontrivial "tricks" are as follows:

(i) When integrating over $\bar{\lambda} \lambda$ to invert the transform (13), it helps to also average over the phase of $\lambda_{\alpha}$, using the known weight of $f^{( \pm s)}$ under such phase rotations. We then have an integral over both magnitudes and phases, which becomes an integral $d z d \bar{z}$ over the complex plane.

(ii) Conversely, when integrating over $\mu^{\alpha}, \bar{\mu}^{\alpha}$ in (17), it helps to localize the integral on values of $\mu^{\alpha}$ with an overall phase given by (19), where $\mathbf{r}^{\prime} \equiv(\bar{\mu} \boldsymbol{\sigma} \mu) /(\bar{\mu} \mu)$ and $\mathbf{m}^{\prime} \equiv(i \mu \boldsymbol{\sigma} \mu) /(\sqrt{2} \bar{\mu} \mu)$.

\section{TWISTORIAL DERIVATION}

To prove our S-matrix formula (17), we will relate it to a picture that is covariant under the full $S O(1,4)$ de Sitter group. First, we will rewrite our transforms (13), (16) between spinor-helicity functions and horizon data in $S O(1,4)$-covariant language. For this, we combine $\lambda_{\alpha}$ and $i \bar{\mu}^{\alpha}$ into a twistor, i.e., an $S O(1,4)$ spinor $Y^{a}$, as in (3). The final horizon's spinor functions (13) can now be treated as functions of the twistor variables $(Y, \bar{Y})$, which just happen to depend only on the $\left(\gamma_{u} Y, \gamma_{u} \bar{Y}\right)$ components, i.e., the ones containing $(\lambda, \bar{\lambda})$

$$
f(Y, \bar{Y})=\int d u e^{i\left(\bar{Y} \gamma_{u} Y\right) u / 2} \phi\left(u, \frac{\bar{Y} \gamma_{u} \gamma Y}{i \bar{Y} \gamma_{u} Y} ; \frac{Y \gamma_{u} \gamma Y}{\sqrt{2} \bar{Y} \gamma_{u} Y}\right),
$$

where we removed the helicity superscripts to save space. The initial horizon's spinor functions (16) are now given by the exact same formulas, but with $u$ replaced everywhere by $v$. Of course, this replacement amounts to interchanging the null axes in $\mathbb{R}^{1,4}$ that define the two horizons.

For the second step of our proof, recall that free massless fields in $d S_{4}$ (or in any conformally flat four-dimensional [4d] spacetime) can be encoded, via the Penrose transform $[21,22]$, as holomorphic twistor functions $F^{( \pm s)}\left(Y^{a}\right)$, with no dependence on the complex conjugate $\bar{Y}^{a}$. The de Sitter group $S O(1,4)$ [and, in fact, the entire conformal group $S O(2,4)]$ is realized on these functions by linear transformations of the twistor argument $Y^{a}$. In particular, the Poincare-patch translations $u \rightarrow u-2 \mathbf{a} \cdot \mathbf{r}$ and dilatations $(u, v) \rightarrow\left(e^{t} u, e^{-t} v\right)$ are realized, respectively, as

$$
\begin{aligned}
& F^{( \pm s)}\left(\lambda_{\alpha}, i \bar{\mu}^{\alpha}\right) \rightarrow F^{( \pm s)}\left(\lambda_{\alpha}, i \bar{\mu}^{\alpha}+i(\mathbf{a} \cdot \boldsymbol{\sigma})^{\alpha \beta} \lambda_{\beta}\right), \\
& F^{( \pm s)}\left(\lambda_{\alpha}, i \bar{\mu}^{\alpha}\right) \rightarrow F^{( \pm s)}\left(e^{t / 2} \lambda_{\alpha}, i e^{-t / 2} \bar{\mu}^{\alpha}\right),
\end{aligned}
$$

along with the obvious action of $S O(3)$ rotations. Finally, the field's helicity is encoded in the twistor function's degree of homogeneity,

$$
\lambda_{\alpha} \frac{\partial F^{( \pm s)}}{\partial \lambda_{\alpha}}+\bar{\mu}^{\alpha} \frac{\partial F^{( \pm s)}}{\partial \bar{\mu}^{\alpha}}=Y^{a} \frac{\partial F^{( \pm s)}}{\partial Y^{a}}=-2 \pm 2 s .
$$

We can now see that the Fourier transform of the twistor function $F^{( \pm s)}(\lambda, i \bar{\mu})$ with respect to $\bar{\mu}^{\alpha}$ has the defining symmetries of the spinor-helicity function $f^{( \pm s)}(\lambda, \bar{\lambda})$ on the final horizon! Therefore, up to a prefactor that can be absorbed into the definition of $F^{( \pm s)}(\lambda, i \bar{\mu})$, we identify

$$
f^{( \pm s)}(\lambda, \bar{\lambda})=\int \frac{d^{2} \bar{\mu}}{2 \pi} F^{( \pm s)}(\lambda, i \bar{\mu}) e^{i \bar{\lambda} \bar{\mu}}
$$

or, in $S O(1,4)$-covariant notation

$$
f(Y, \bar{Y})=\int \frac{d Z \gamma_{v} d Z}{4 \pi} F\left(\gamma_{v} \gamma_{u} Y+\gamma_{u} \gamma_{v} Z\right) e^{i \bar{Y} \gamma_{u} \gamma_{v} Z}
$$

where the helicity superscripts are again omitted. But now, by $S O(1,4)$ covariance, upon interchanging $u \leftrightarrow v$, we must get the spinor-helicity functions $\tilde{f}^{( \pm s)}(Y, \bar{Y})$ for the initial horizon! Back in $S O(3)$ spinor notation, this last statement reads

$$
\tilde{f}^{( \pm s)}(\mu, \bar{\mu})=-\int \frac{d^{2} \lambda}{2 \pi} F^{( \pm s)}(\lambda, i \bar{\mu}) e^{-i \lambda \mu}
$$

Combining the two "half"-Fourier transforms (22) and (23), we obtain the free S-matrix (17). 


\section{CONCLUSION}

In this paper, we applied a spinor-helicity formalism to the horizon data of massless fields in de Sitter space and derived an elegant expression for the free S-matrix of massless fields with any spin. We extended the known analogies [7] with the spinor-helicity formalism in flat spacetime, in particular establishing a "half"-Fourier transform relation between spinor-helicity and twistor functions. We also went beyond [7] by working with two different horizons, each of which has a different notion of momentum. Despite this seeming complication, we saw that the momenta on the two horizons are related by a simple Fourier transform (17), once we consider their spinor square root. This Fourier-transform relationship can also be understood as a change of basis in the Hilbert space of a massless particle on the (complexified) $3 \mathrm{~d}$ conformal boundary [12].
Having found the free S-matrix in the spinor-helicity basis, we obtained an explicit expression (18) in terms of horizon field data for any spin. More importantly, there is now hope that perturbation theory for interacting fields in the de Sitter causal patch can be constructed with relative ease. In the future, we would like to explore this possibility, in particular for Yang-Mills and GR. In addition, we expect that the language developed here can be fruitfully applied to higher-spin gravity $[23,24]$, the only known working model [25] for dS/CFT holography in $3+1$ dimensions.

\section{ACKNOWLEDGMENTS}

This work was supported by the Quantum Gravity and Mathematical and Theoretical Physics Units of the Okinawa Institute of Science and Technology Graduate University. N. F.'s work was carried out during an internship at OIST.
[1] R. Kleiss and W. J. Stirling, Spinor techniques for calculating $p \bar{p} \rightarrow W^{ \pm} / Z^{0}+$ jets, Nucl. Phys. B262, 235 (1985).

[2] L. J. Dixon, A brief introduction to modern amplitude methods, arXiv:1310.5353.

[3] L. J. Mason and D. Skinner, Scattering amplitudes and BCFW recursion in twistor space, J. High Energy Phys. 01 (2010) 064.

[4] N. Arkani-Hamed, F. Cachazo, C. Cheung, and J. Kaplan, The S-Matrix in twistor space, J. High Energy Phys. 03 (2010) 110.

[5] N. Arkani-Hamed, J. L. Bourjaily, F. Cachazo, A. B. Goncharov, A. Postnikov, and J. Trnka, Grassmannian geometry of scattering amplitudes, arXiv:1212.5605.

[6] J. M. Maldacena, Non-Gaussian features of primordial fluctuations in single field inflationary models, J. High Energy Phys. 05 (2003) 013.

[7] J. M. Maldacena and G. L. Pimentel, On graviton nonGaussianities during inflation, J. High Energy Phys. 09 (2011) 045.

[8] N. Arkani-Hamed, P. Benincasa, and A. Postnikov, Cosmological polytopes and the wavefunction of the Universe, arXiv: 1709.02813 .

[9] M. Plyushchay, D. Sorokin, and M. Tsulaia, Higher spins from tensorial charges and $\mathrm{OSp}(\mathrm{N}-2 \mathrm{n})$ symmetry, J. High Energy Phys. 04 (2003) 013.

[10] B. Nagaraj and D. Ponomarev, Spinor-Helicity Formalism for Massless Fields in $\mathrm{AdS}_{4}$, Phys. Rev. Lett. 122, 101602 (2019).

[11] Y. Neiman, Twistors and antipodes in de Sitter space, Phys. Rev. D 89, 063521 (2014).

[12] Y. Neiman, Holographic quantization of linearized higherspin gravity in the de Sitter causal patch, J. High Energy Phys. 11 (2018) 033.

[13] C. Fronsdal, Singletons and massless, integral spin fields on de Sitter space (elementary particles in a curved space. 7.), Phys. Rev. D 20, 848 (1979).
[14] D. J. Pritchard and W. J. Stirling, QCD calculations in the light cone gauge. 1, Nucl. Phys. B165, 237 (1980).

[15] A. K. H. Bengtsson, I. Bengtsson, and L. Brink, Cubic interaction terms for arbitrary spin, Nucl. Phys. B227, 31 (1983).

[16] D. Ponomarev and E. D. Skvortsov, Light-front higher-spin theories in flat space, J. Phys. A 50, 095401 (2017).

[17] R. Penrose, Null hypersurface initial data for classical fields of arbitrary spin and for general relativity, Gen. Relativ. Gravit. 12, 225 (1980).

[18] L. Hackl and Y. Neiman, Horizon complementarity in elliptic de Sitter space, Phys. Rev. D 91, 044016 (2015).

[19] I. F. Halpern and Y. Neiman, Holography and quantum states in elliptic de Sitter space, J. High Energy Phys. 12 (2015) 057.

[20] R. Britto, F. Cachazo, B. Feng, and E. Witten, Direct Proof of Tree-Level Recursion Relation in Yang-Mills Theory, Phys. Rev. Lett. 94, 181602 (2005).

[21] R. Penrose and W. Rindler, Spinors and Space-Time. Vol. 2: Spinor and Twistor Methods in Space-Time Geometry (Cambridge University Press, Cambridge, United Kingdom, 1986), p. 501.

[22] R. S. Ward and R. O. Wells, Twistor Geometry and Field Theory (Cambridge University Press, Cambridge, United Kingdom, 1990), p. 520.

[23] M. A. Vasiliev, Higher spin gauge theories in four-dimensions, three-dimensions, and two-dimensions, Int. J. Mod. Phys. D 05, 763 (1996).

[24] M. A. Vasiliev, Higher spin gauge theories: Star product and AdS space, in The Many Faces of the Superworld, edited by M. A. Shifman (World Scientific, Singapore, 2010), pp. 533-610.

[25] D. Anninos, T. Hartman, and A. Strominger, Higher spin realization of the dS/CFT correspondence, Classical Quantum Gravity 34, 015009 (2017). 